\title{
Dynamic Model and Vibration Characteristics of Planar 3-RRR Parallel Manipulator with Flexible Intermediate Links considering Exact Boundary Conditions
}

\author{
Lianchao Sheng, Wei Li, Yuqiao Wang, Mengbao Fan, and Xuefeng Yang \\ School of Mechatronic Engineering, China University of Mining and Technology, Xuzhou 221116, China \\ Correspondence should be addressed to Wei Li; cmeecumt512@yahoo.com
}

Received 11 January 2017; Revised 28 March 2017; Accepted 24 May 2017; Published 12 July 2017

Academic Editor: Tai Thai

Copyright (c) 2017 Lianchao Sheng et al. This is an open access article distributed under the Creative Commons Attribution License, which permits unrestricted use, distribution, and reproduction in any medium, provided the original work is properly cited.

Due to the complexity of the dynamic model of a planar 3-RRR flexible parallel manipulator (FPM), it is often difficult to achieve active vibration control algorithm based on the system dynamic model. To establish a simple and efficient dynamic model of the planar 3-RRR FPM to study its dynamic characteristics and build a controller conveniently, firstly, considering the effect of rigidflexible coupling and the moment of inertia at the end of the flexible intermediate link, the modal function is determined with the pinned-free boundary condition. Then, considering the main vibration modes of the system, a high-efficiency coupling dynamic model is established on the basis of guaranteeing the model control accuracy. According to the model, the modal characteristics of the flexible intermediate link are analyzed and compared with the modal test results. The results show that the model can effectively reflect the main vibration modes of the planar 3-RRR FPM; in addition the model can be used to analyze the effects of inertial and coupling forces on the dynamics model and the drive torque of the drive motor. Because this model is of the less dynamic parameters, it is convenient to carry out the control program.

\section{Introduction}

Compared with the serial manipulator, the parallel manipulator has the advantages of high speed, high acceleration, high load capacity, low energy consumption, no-accumulation error, and high precision, which is widely used in aerospace, precision optical instruments, high-precision real-time measuring instruments, fast precision machine tools, and other fields [1]. Simultaneously, FPMs have attracted great social concerns and have been applied extensively. However, owning to the inertia force, dynamic load carrying capacity, and other factors, flexible intermediate links are prone to elastic deformation, which results in the decrease of the overall dynamic performance of the manipulator and influences the normal working of the FPM under the high speed with load condition. Elastic vibration has a greater impact on the accuracy and quality of the operation in the system $[2,3]$, for example, the elastic vibration of pick-and-place parallel manipulator in food packaging, directly affecting the quality of food [4]; the vibration of operating mechanism at the end of picking manipulator, leading to instability in grasping and damage to strawberry, tomatoes, and other soft appearance fruits $[5,6]$. Therefore, the study of vibration characteristics of the FPM has important significance.

In order to further study the vibration problem of the planar 3-RRR FPM with flexible intermediate links, the dynamic model needs to be established which can accurately reflect the rigid-flexible coupling characteristics of the system. Furthermore, the vibration characteristics and the influences should be studied between the rigid body and the flexible body based on the model. In the existing literatures, the dynamic modeling method of the FPM is mainly from the energy point of view to obtain the partial differential equation. Then an approximation finite-dimensional ordinary differential equation is obtained by discretizing the complex continuous system model through the finite element method (FEM) $[7,8]$ and the AMM $[9,10]$. Yu and Hong [11] described dynamic modeling, modal selection and modal synthesis, and dynamic stiffening of flexible multibody systems. Yu et al. [12] presented a dynamic model of the 3-RRR FPM and 
verified the correctness of the model through experiments. Zhang et al. $[13,14]$ established the dynamic model of the 3-RRR FPM using the finite element method and Lagrange equation and analyzed the influence of temperature and other factors on the elastic deformation of each flexible link. Zhang et al. [15] established the dynamic model of the 3-RRR FPM based on the energy method and illustrated the influence of the joint gap on the model. Fattah et al. [16] studied the dynamics of a 3-DOF parallel manipulator. Based on the finite element method and the Euler-Lagrange equation, the dynamic equations of the system were established. In order to emphasize the influence of system flexibility, the dynamics simulation experiment of rigid-flexible system was carried out. In spite of the high precision of the above models, the dynamic model described in the past literature has too many dynamic parameters, which makes the model difficult to solve and is disadvantageous to the design of subsequent controllers. In order to meet the requirements of the model premise, as far as possible to make the model simplification, low-level, easy to solve, and controller design, Mostafavi Yazdi and Irani [17] described the basic principle and modeling process of the AMM in detail and investigated the dynamic equations of the beam model with two teeth which were established by the AMM. Compared with the general beam model, the elastic vibration of the tooth beam is smaller. Chen [18] established a linear dynamic model of a multiflexible linkage manipulator by Lagrange equation and AMM. During the dynamic modeling of planar FPM with flexible intermediate links, Zhang et al. [19, 20] elaborated the dynamic model of FPM with flexible intermediate links which was established using the boundary conditions of pinned-pinned at both ends of the flexible intermediate links based on AMM and the Lagrange equation. However, the experiment in $[21,22]$ proved that, in the motion control, because of the elastic deformation of the flexible link, the dynamic platform produced violent vibration. It can not explain this phenomenon well using pinned-pinned boundary conditions vibration mode to discretize the flexible deformation.

Different from current literatures, in this paper, based on the extended Hamilton principle and AMM, with the pinned-free boundary condition for the flexible intermediate link, the dynamic model of the 3-RRR FPM with flexible intermediate links is established with considering the effect of rotor and the concentrated moment of inertia at both ends of link and the rigid-flexible coupling. The dynamic model established can not only satisfy the model precision but also solve the dynamic model easily. The dynamic response and the natural frequency of the flexible intermediate link are obtained by solving the dynamic equation. Based on the model, the influence of the coupling force and inertial force on the system and the driving torque of the motor are analyzed. Finally, the correctness of the model is verified by modal experiment. This provides a theoretical basis for the optimal design of the system structure and shock absorbers and the selection of motors.

The structure of the paper is as follows. The second chapter introduces the system overview and dynamic modeling of the planar 3-RRR FPM. The numerical simulations and

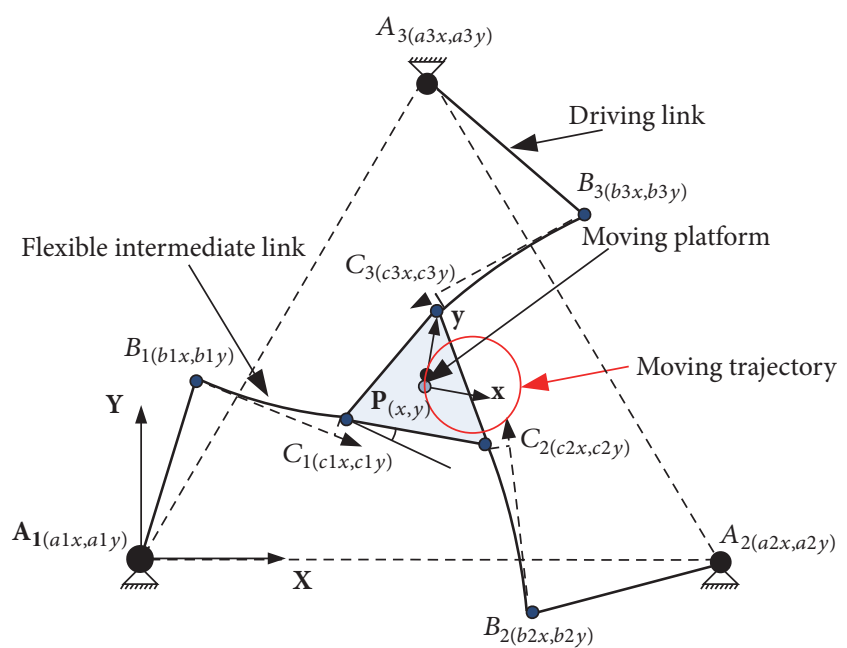

FIgURE 1: The structure diagram of the planar 3-RRR FPM.

results discussions are shown in third chapter. The forth chapter is the experimental verification section. Finally, the paper is concluded with a brief summary.

\section{The System Overview and Dynamic Modeling of the Planar 3-RRR FPM}

The system structure and the coordinate system of the planar 3-RRR FPM are shown in Figure 1. $X A_{1} Y$ is the global coordinate system. $x P y$ is the moving coordinate system which follows the moving platform. The system is composed of the moving platform $C_{1} C_{2} C_{3}$ and the fixed base $A_{1} A_{2} A_{3}$ as well as three identical branches $A_{1} B_{1} C_{1}, A_{2} B_{2} C_{2}$, and $A_{3} B_{3} C_{3}$. As Figure 1 shows, $A_{1}, A_{2}$, and $A_{3}$ are the three vertices of the regular triangle whose side length is $500 \mathrm{~mm}$, where the active revolute joints are set. The points of $B_{1}$, $C_{1}, B_{2}, C_{2}, B_{3}$, and $C_{3}$ are the passive revolute joints, where $A_{1} B_{1}=A_{2} B_{2}=A_{3} B_{3}=B_{1} C_{1}=B_{2} C_{2}=B_{3} C_{3}=L_{i}=$ $210 \mathrm{~mm}$. The radius of the circle of joints in moving platform is $R=68 \mathrm{~mm}$. The 3-DOF planar motion can be achieved by using the three driving motors in the three active joints.

In the actual structure, the cross-sectional area of the drive rod is larger to ensure its rigidity, while the crosssectional area of the intermediate link is smaller to reduce its mass. Therefore, in this paper, only the flexible intermediate links of the flexible planar 3-RRR parallel robots are considered as the flexible part; others are the rigid part. The vibration of the mechanism is mainly caused by the motor drive, the inertial force, and the payload of the moving platform.

2.1. Dynamic Modeling of Flexible Intermediate Link. The schematic diagram of the $i$ th branch and moving platform are shown in Figure 2. The transverse deformation is smaller compared with the length of the flexible intermediate link, so the equation can be modeled linearly according to the linear elastic deformation. The flexible intermediate link is considered as a pinned-free Euler-Bernoulli beam. The 

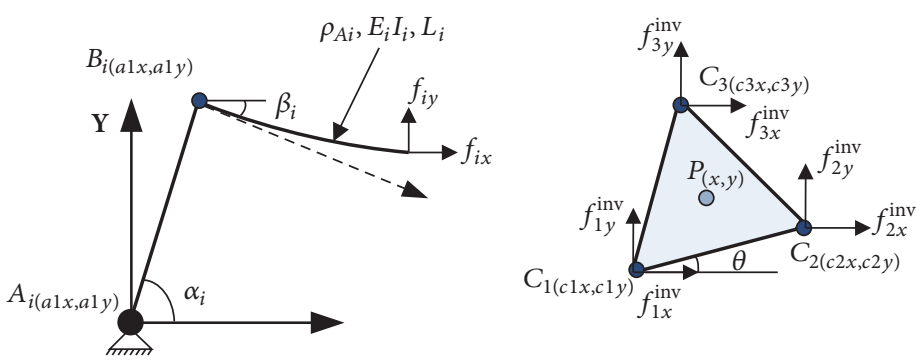

FIGURE 2: Schematic diagram of the $i$ th branch and moving platform.

radial and lateral stiffness is ignored, and the uncertainty of modeling and other parameters is regarded as a kind of parameter perturbation. Since the flexible intermediate link moves in the horizontal plane, the effect of gravity is not considered. The kinetic energy mainly includes the rotational kinetic energy of the motor rotor and the rotational kinetic energy of the joint $A_{i}$, the translational kinetic energy of driving rod, the concentrated translational kinetic energy of the joint $B_{i}$ and its own rotational kinetic energy, and the translational kinetic energy of the intermediate link, where the rotational kinetic energy of joints $A_{i}$ and $B_{i}$ is mainly produced by its internal bearing. It can be expressed as

$$
T_{i}=T_{A_{i}}+T_{A_{i} B_{i}}+2 T_{B_{i}}+T_{B_{i} C_{i}} \quad i=1,2,3,
$$

where $T_{A_{i}}=(1 / 2) J_{A_{i}} \dot{\alpha}_{i}^{2}$ is the rotational kinetic energy of the $i$ th drive joint $A_{i}$ and the motor rotor; $T_{A_{i} B_{i}}=$ $(1 / 2) \int_{0}^{L_{i}} \rho_{A_{i}} \dot{\vec{r}}_{i}^{2} d x$ is the kinetic energy of the $i$ th driving rod; $T_{B_{i}}=(1 / 2) m_{B_{i}} \dot{\vec{r}}_{B_{i}}^{2}+(1 / 2) J_{B_{i}}\left(\dot{\alpha}+\dot{w}_{B_{i}}^{\prime}\right)^{2}$ is the kinetic energy of the joint $B_{i} ; T_{B_{i} C_{i}}=(1 / 2) \int_{0}^{L_{i}} \rho_{A_{i}} \dot{\vec{k}}_{i}^{2} d x$ is the kinetic energy of the $i$ th flexible intermediate link; $w_{i}\left(x_{i}, t\right)$ is the transverse elastic displacement of $x$ points on flexible intermediate link; $\rho_{A_{i}}$ is the unit length mass of the $i$ th flexible intermediate link; $m_{B_{i}}$ is the mass of the joint $B_{i} ; J_{A_{i}}$ is the rotational inertia of the driving joint $A_{i}$ and the motor rotor; $J_{B_{i}}$ is the rotational inertia of joint $B_{i} ; \vec{r}_{i}=\left[x_{a i}+x_{i} \cos \alpha_{i}\right] i+\left[y_{a i}+x_{i} \sin \alpha_{i}\right] j$ is the vector coordinates of the $i$ th drive rod; $\vec{k}_{i}=\left[x_{a i}+\right.$ $\left.L_{i} \cos \alpha_{i}+x_{i} \cos \beta_{i}-w\left(x_{i}, t\right) \sin \beta_{i}\right] i+\left[y_{a i}+L_{i} \sin \alpha_{i}+x_{i} \sin \beta_{i}+\right.$ $\left.w\left(x_{i}, t\right) \cos \beta_{i}\right] j$ is the vector coordinates of the $i$ th flexible intermediate link; $(\dot{w})$ and $\left(w^{\prime}\right)$ are the differential for the time and displacement, respectively.

The potential energy of the $i$ th branch of the system is expressed as

$$
V_{i}=\frac{1}{2} \int_{0}^{L_{i}} E_{i} I\left(x_{i}\right)\left(w^{\prime \prime}\right)^{2} d x_{i},
$$

where $E_{i}$ is the elastic modulus and $I\left(x_{i}\right)$ is the moment of inertia of section of flexible intermediate link.

The virtual work of the $i$ th branch of the system can be expressed as

$$
\delta W_{i}=\bar{f}_{i x y} \cdot \delta \vec{k}_{C_{i}}+M_{A_{i}} \delta \alpha_{i}
$$

where $\bar{f}_{i x y}=\left[\begin{array}{ll}f_{i x} & f_{i y}\end{array}\right]$ is the constraint force between the joint $C_{i}$ and the end of the flexible intermediate link. $M_{A_{i}}$ is the drive torque of the $i$ th servomotor. $\delta \vec{k}_{C_{i}}=\left.\delta \vec{k}_{i}\right|_{x=L_{i}}$ is the virtual displacement vector at the end of the $i$ th flexible intermediate link. $\delta \alpha_{i}$ is the virtual angle displacement of the $i$ th servomotor.

Based on the extend Hamilton principle [23], the boundary conditions and the kinetic differential equation can be derived as follows:

$$
\int_{t 1}^{t 2}\left(\delta T_{i}-\delta V_{i}+\delta W_{i}\right) d t=0, \quad \delta \bar{\eta}_{i}(t 1)=\delta \bar{\eta}_{i}(t 2)=0,
$$

where $\bar{\eta}_{i}=\left[\begin{array}{lll}\alpha_{i} & \beta_{i} & w_{i}\end{array}\right]^{T}$ is the generalized variable of the $i$ th branch.

According to the extended Hamilton principle, the total energy of the $i$ th branch is varied to the generalized variable. Considering the interchangeability between variational and differential, the similar items are combined after integrating the variational components by fractional integration over time $t 1$ to $t 2$. Since $\delta \alpha_{i}, \delta \beta_{i}, \delta w_{i}, \delta w_{B_{i}}, \delta w_{B_{i}}^{\prime}, \delta w_{C_{i}}, \delta w_{C_{i}}^{\prime}$ can be assigned any value, three differential equations for generalized variables and four boundary conditions for flexible intermediate links are obtained. Since the flexible intermediate link is pinned to joint $B_{i}$, the deformation of the pinned joint is zero according to $[24,25]$. This conclusion is used to simplify the differential equation.

The differential equation about $\delta \alpha_{i}$ is as follows:

$$
\begin{aligned}
& M_{A_{i}}+f_{i y} L_{i} \cos \alpha_{i}-f_{i x} L_{i} \sin \alpha_{i}=J_{A_{i}} \ddot{\alpha}_{i}+m_{B_{i}} L_{i}^{2} \ddot{\alpha}_{i} \\
& \quad+\int_{0}^{L_{i}} \rho_{A_{i}}\left(L_{i}^{2} \ddot{\alpha}_{i}+x_{i}^{2} \ddot{\alpha}_{i}+S_{i} L_{i} x_{i} \dot{\beta}_{i}^{2}+C_{i} L_{i} x_{i} \ddot{\beta}_{i}\right. \\
& \quad+2 S_{i} L_{i} \dot{\beta}_{i} \dot{w}_{i}+C_{i} L_{i} \ddot{w}_{i}+S_{i} L_{i} w_{i} \ddot{\beta}_{i} \\
& \left.\quad-C_{i} L_{i} w_{i} \dot{\beta}_{i}^{2}\right) d x_{i} .
\end{aligned}
$$

The differential equation about $\delta \beta_{i}$ is as follows:

$$
\begin{aligned}
& \left(f_{i y} L_{i} \cos \beta_{i}-f_{i y} w_{C_{i}} \sin \beta_{i}-f_{i x} L_{i} \sin \beta_{i}-f_{i x} w_{C_{i}}\right. \\
& \left.\cdot \cos \beta_{i}\right)=J_{B_{i}} \ddot{\beta}_{i}+\int_{0}^{L_{i}} \rho_{A_{i}}\left(x_{i}^{2} \ddot{\beta}_{i}+2 w_{i} \dot{w}_{i} \dot{\beta}_{i}+w_{i}^{2} \ddot{\beta}_{i}\right. \\
& \quad-S_{i} L_{i} x_{i} \ddot{\alpha}_{i}+C_{i} L_{i} x_{i} \ddot{\alpha}_{i}+C_{i} L_{i} w_{i} \dot{\alpha}_{i}^{2}+S_{i} L_{i} w_{i} \ddot{\alpha}_{i} \\
& \left.\quad+x_{i} \ddot{w}_{i}\right) d x_{i} .
\end{aligned}
$$


The differential equation about $\delta w_{i}$ is as follows:

$$
\begin{aligned}
& \rho_{A_{i}}\left(\ddot{w}_{i}-\dot{\beta}_{i}^{2} w_{i}-S_{i} L_{i} \dot{\alpha}_{i}+C_{i} L_{i} \ddot{\alpha}_{i}+x_{i} \ddot{\beta}_{i}\right)+E_{i} I_{i} w^{\prime \prime \prime \prime} \\
& \quad=0 .
\end{aligned}
$$

The four nonhomogeneous boundary conditions of the flexible intermediate link are as follows:

$$
\begin{aligned}
w_{i}(0, t) & =0, \\
E_{i} I_{i} w_{i}^{\prime \prime}(0, t) & =J_{B_{i}}\left[\ddot{\beta}_{i}+\ddot{w}_{i}^{\prime}(0, t)\right], \\
E_{i} I_{i} w_{i}^{\prime \prime}\left(L_{i}, t\right) & =0, \\
E_{i} I_{i} w_{i}^{\prime \prime \prime}\left(L_{i}, t\right) & =f_{i y} \cos \beta_{i}-f_{i x} \sin \beta_{i} .
\end{aligned}
$$

In order to obtain the homogeneous boundary condition for flexible deformation discrete based on AMM, the Dirac function deformation is introduced according to [22].

$$
\begin{aligned}
\delta\left(x_{i}-L_{i}\right) & =0 \quad x_{i} \neq L_{i}, \\
\int_{0}^{L_{i}} \delta\left(x_{i}-L_{i}\right) d x_{i} & =1 .
\end{aligned}
$$

Substituting (9) into (7) and (8),

$$
\begin{aligned}
& \rho_{A_{i}}\left(\ddot{w}_{i}-\dot{\beta}_{i}^{2} w_{i}-S_{i} L_{i} \dot{\alpha}_{i}+C_{i} L_{i} \ddot{\alpha}_{i}+x_{i} \ddot{\beta}_{i}\right)+E_{i} I_{i} w^{\prime \prime \prime \prime} \\
& \quad=\left(f_{i y} \cos \beta_{i}-f_{i x} \sin \beta_{i}\right) \delta\left(x_{i}-L_{i}\right) \\
& \quad+J_{B_{i}}\left[\ddot{\beta}_{i}+\ddot{w}_{i}^{\prime}(0, t)\right] \delta^{\prime}\left(x_{i}\right), \\
& w_{i}(0, t)=0, \\
& E_{i} I_{i} w_{i}^{\prime \prime}(0, t)=0, \\
& E_{i} I_{i} w_{i}^{\prime \prime}\left(L_{i}, t\right)=0, \\
& E_{i} I_{i} w_{i}^{\prime \prime \prime}\left(L_{i}, t\right)=0 .
\end{aligned}
$$

As can be seen from (11), the deflection and bending moment at the joint $B_{i}$ are zero, and the bending moments and shear forces at the joint $C_{i}$ are zero. Therefore, the pinned-free boundary conditions are satisfied for the flexible intermediate link.

2.2. Elastic Deformation Discretization of Flexible Intermediate Link. After the above analysis, we can find that the established equation is a highly nonlinear differential equation with rigid motion and elastic motion coupling, and the analytical solution is difficult. Therefore, it is considered to use the AMM to discretize the elastic deformation of the flexible link and then to solve the numerical solution for dynamic analysis. According to AMM, the deformation of the flexible intermediate link can be expressed as follows:

$$
w_{i}\left(x_{i}, t\right)=\sum_{j=1}^{r} \varphi_{i j}\left(x_{i}\right) q_{i j}(t),
$$

where $q_{i j}(t)$ denotes the unknown generalized elastic variable of the $i$ th flexible intermediate link. $\varphi_{i j}\left(x_{i}\right)$ is the mode function corresponding to the known boundary condition.

From the analysis of the previous section, the mode shape function with pinned-free boundary conditions of the flexible intermediate link is used to discrete elastic deformation. As can be seen from [24], the modal functions under the pinned-free boundary condition are shown as follows:

$$
\begin{aligned}
\varphi_{j}(x) & =\frac{1}{\sqrt{m L} \sin \theta_{j}}\left[\sin \frac{\theta_{j} x}{L}+\frac{\sin \theta_{j}}{\sinh \theta_{j}} \sinh \frac{\theta_{j} x}{L}\right], \\
\theta_{j} & =(j+0.25) \pi, \quad j=1,2,3, \ldots, r .
\end{aligned}
$$

Equations (12) and (13) are substituted into (5), (6), and (7), respectively.

$$
\begin{aligned}
& M_{A_{i}}+f_{i y} L_{i} \cos \alpha_{i}-f_{i x} L_{i} \sin \alpha_{i}=J_{A_{i}} \ddot{\alpha}_{i}+m_{B_{i}} L_{i}^{2} \ddot{\alpha}_{i} \\
& +\int_{0}^{L_{i}} \rho_{A_{i}}\left(L_{i}^{2} \ddot{\alpha}_{i}+x_{i}^{2} \ddot{\alpha}_{i}+S_{i} L_{i} x_{i} \dot{\beta}_{i}^{2}+C_{i} L_{i} x_{i} \ddot{\beta}_{i}\right. \\
& +2 S_{i} L_{i} \dot{\beta}_{i} \sum_{j=1}^{r} \varphi_{i j}\left(x_{i}\right) \dot{q}_{i j}(t)+C_{i} L_{i} \sum_{j=1}^{r} \varphi_{i j}\left(x_{i}\right) \ddot{q}_{i j}(t) \\
& +S_{i} L_{i} \ddot{\beta}_{i} \sum_{j=1}^{r} \varphi_{i j}\left(x_{i}\right) q_{i j}(t) \\
& \left.+C_{i} L_{i} \sum_{j=1}^{r} \varphi_{i j}\left(x_{i}\right) q_{i j}(t) \dot{\beta}_{i}^{2}\right) d x_{i}, \\
& +f_{i y} L_{i} \cos \beta_{i}^{2}-f_{i j} w_{C_{i}} \sin \beta_{i}-f_{i x} L_{i} \sin \beta_{i}-f_{i x} w_{C_{i}} \\
& +E_{i} I_{i} \int_{0}^{L_{i}} \varphi_{i j}^{\prime \prime 2}\left(x_{i}\right) d x_{i}-\dot{\beta}_{i}^{2} \int_{0}^{L_{i}} \rho_{A_{i}} \varphi_{i j}^{2}\left(x_{i}\right) d x_{i} \ddot{q}_{i j}(t) \ddot{q}_{i j}(t) \\
& +C_{i} L_{i} \dot{\alpha}_{i}^{2} \sum_{j=1}^{r} \varphi_{i j}\left(x_{i}\right) q_{i j}(t)+S_{i} L_{i} \sum_{j=1}^{r} \varphi_{i j}\left(x_{i}\right) q_{i j}(t) \ddot{\alpha}_{i} \\
& +\left(\sum_{j=1}^{r} \varphi_{i j}\left(x_{i}\right) q_{i j}(t) \ddot{\beta}_{i}-S_{i} L_{i} x_{i} \ddot{\alpha}_{i}+C_{i} L_{i} x_{i} \ddot{\alpha}_{i}\right. \\
& +2\left(\sum_{j=1}^{L_{i}} \varphi_{i j}\left(x_{i}\right) q_{i j}(t)\right)\left(\sum_{B_{i}}^{2} \ddot{\beta}_{i} \varphi_{i j}\left(x_{i}\right) \dot{q}_{i j}(t)\right) \dot{\beta}_{i} \\
& \quad+1
\end{aligned}
$$

$$
0 \leq x_{i} \leq L_{i}, \quad i=1,2,3,
$$




$$
\begin{aligned}
& \cdot q_{i j}(t)=\int_{0}^{L_{i}} \rho_{A_{i}}\left(C_{i} L_{i} \ddot{\alpha}-S_{i} L_{i} \dot{\alpha}\right) \varphi_{i j}\left(x_{i}\right) d x_{i} \\
& -\ddot{\beta}\left[\int_{0}^{L_{i}} \rho_{A_{i}} x_{i} \varphi_{i j}\left(x_{i}\right) d x_{i}+J_{B_{i}} \varphi_{i j}^{\prime 2}(0)\right]+\varphi_{i j}\left(L_{i}\right) \\
& \cdot\left(f_{i y} \cos \beta_{i}-f_{i x} \sin \beta_{i}\right) .
\end{aligned}
$$

2.3. Dynamic Model of Moving Platform. The moving platform is considered to be a rigid body in the modeling process. The dynamic equation of the moving platform is as follows:

$$
\begin{aligned}
& {\left[\begin{array}{ccc}
m_{p} & 0 & 0 \\
0 & m_{p} & 0 \\
0 & 0 & J_{\varphi p}
\end{array}\right]\left[\begin{array}{l}
\ddot{x}_{p} \\
\ddot{y}_{p} \\
\ddot{\varphi}_{p}
\end{array}\right]} \\
& =\left[\begin{array}{cccccc}
-1 & 0 & -1 & 0 & -1 & 0 \\
0 & -1 & 0 & -1 & 0 & -1 \\
e_{1 y} & -e_{1 x} & e_{2 y} & -e_{2 x} & e_{3 y} & -e_{3 x}
\end{array}\right]\left[\begin{array}{l}
f_{1 x} \\
f_{1 y} \\
f_{2 x} \\
f_{2 y} \\
f_{3 x} \\
f_{3 y}
\end{array}\right],
\end{aligned}
$$

where $\bar{e}_{i}=\left(e_{1 x}, e_{1 y}\right)=\left(x_{c i}^{\prime} \cos \varphi_{p}-y_{c i}^{\prime} \sin \varphi_{p}, x_{c i}^{\prime} \sin \varphi_{p}-\right.$ $\left.y_{c i}^{\prime} \cos \varphi_{p}\right)$ and $m_{p}$ is the quality of the moving platform. $J_{\varphi p}$ is the moment of inertia of the moving platform. $\left(x_{c i}^{\prime}, y_{c i}^{\prime}\right)$ is the coordinate of the joint $C_{i}$ in the local coordinate system.

2.4. Rigid-Flexible Coupling Dynamics Model. Combining the dynamic equation of the moving platform with the kinetic equation of the branch, the rigid-flexible coupling ordinary differential equation of the 3-RRR FPM can be obtained as follows:

$$
M \ddot{\bar{\eta}}+C \dot{\bar{\eta}}+K \bar{\eta}=\bar{P}+J_{f} \bar{f},
$$

where $\bar{\eta}=\left[\begin{array}{llll}\bar{\alpha} & \bar{\beta} & \bar{X}_{P} & \bar{q}\end{array}\right]^{T} \in R^{(9+3 r) \times 1}$ is the generalized coordinate variable. $\bar{\alpha}=\left[\begin{array}{lll}\alpha_{1} & \alpha_{2} & \alpha_{3}\end{array}\right]^{T}, \bar{\beta}=\left[\begin{array}{lll}\beta_{1} & \beta_{2} & \beta_{3}\end{array}\right]^{T}$, $\bar{X}_{P}=\left[\begin{array}{lll}x_{p} & y_{p} & \varphi_{p}\end{array}\right]^{T}$ is the rigid body motion coordinate. $\bar{q}=\left[\begin{array}{lllll}q_{11} \cdots q_{1 r} & q_{21} \cdots q_{2 r} & q_{31} \cdots q_{3 r}\end{array}\right]^{T} \in R^{3 r \times 1}$ is the elastic coordinate of three flexible intermediate links. $\bar{P}=$ $\left[\begin{array}{lllll}M_{A_{1}} & M_{A_{2}} & M_{A_{3}} & 0 & \cdots\end{array}\right]^{T} \in R^{(9+3 r) \times 1}$ represents the driving torque of the three motors. $J_{f}$ is the binding matrix. The detailed form of the matrix is shown in Appendix.

$\bar{f}=\left[\begin{array}{llllll}f_{1 x} & f_{1 y} & f_{2 x} & f_{2 y} & f_{3 x} & f_{3 y}\end{array}\right]^{T} \in R^{6 \times 1}$ is the constraints of internal force of the joint $C_{i}$. $M$ is a positive definite symmetric mass matrix. $C$ is the centrifugal force and Coriolis matrix. $K$ is the stiffness matrix.

\section{Numerical Simulation}

The specific material and dimension parameters of the planar 3-RRR FPM are as follows: the material is aluminum alloy
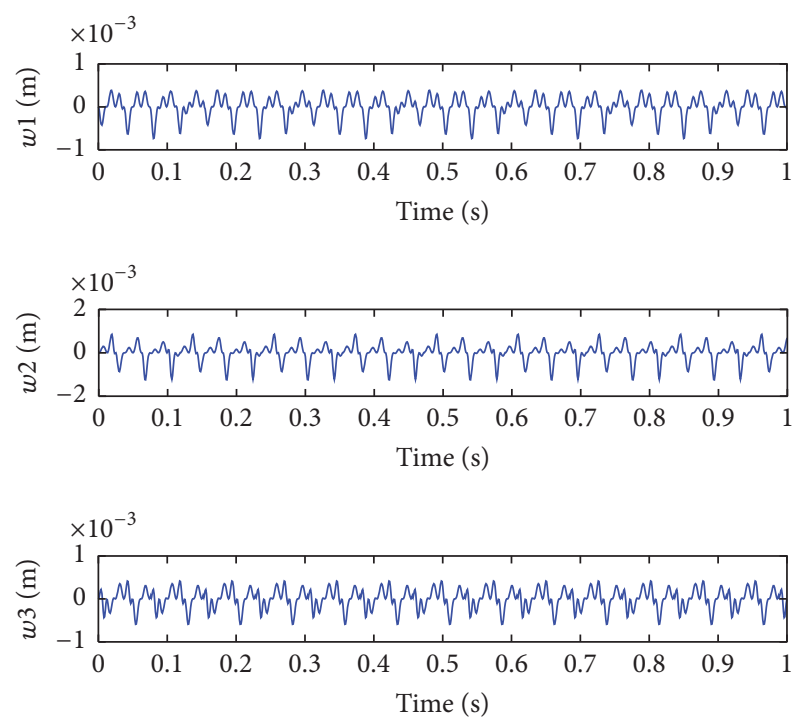

FIGURE 3: Vibration response curves of the end of the flexible intermediate links.

7075. The elastic modulus of the material is $E_{i}=7.1 \times$ $10^{10} \mathrm{~N} / \mathrm{m}^{2}$. The density is $\rho_{i}=2.81 \times 10^{3} \mathrm{~kg} / \mathrm{m}^{3}$. The length, width and thick of the drive rod are $210 \mathrm{~mm}, 25 \mathrm{~mm}$, and $10 \mathrm{~mm}$, respectively. The length, width, and thick of the flexible intermediate link are $210 \mathrm{~mm}, 25 \mathrm{~mm}$, and $2 \mathrm{~mm}$, respectively. The moment of inertia of the motor rotor and drive joint is the $J_{A_{i}}=8.3 \times 10^{-3} \mathrm{~kg} / \mathrm{m}^{2}$. The concentrated moment of inertia at the end of flexible intermediate link is $J_{B_{i}}=2.2 \times 10^{-3} \mathrm{~kg} / \mathrm{m}^{2}$. The moment of inertia of section of flexible intermediate link is $I\left(x_{i}\right)=1.67 \times 10^{-11} \mathrm{~m}^{4}$.

In order to simulate the end of the operating conditions, a typical circular equation is given for its trajectory as follows:

$$
\begin{aligned}
& x_{p}=0.31-0.06 \cos (50 \pi t)(m), \\
& y_{p}=0.25 \sqrt{3}+0.06 \sin (50 \pi t)(m) .
\end{aligned}
$$

The numerical simulation results are as follows.

Figure 3 shows that the amplitude of the first-order end vibration response of the flexible intermediate link is larger. This vibration is sufficient to affect the stability and dynamic performance of the system. Therefore, with the flexible intermediate link 1 as an example, the secondorder vibration response and frequency characteristics are further studied. The amplitude-frequency characteristics can be obtained through fast Fourier transform. The results are shown in Figures 4 and 5.

As shown in Figure 4, the fast Fourier transform of the first-order response curve shows that the frequency components are mainly $24.88 \mathrm{~Hz}, 49.75 \mathrm{~Hz}$, and $104.5 \mathrm{~Hz}$. Through the same treatment, the second-order frequency components are mainly $24.88 \mathrm{~Hz}, 49.75 \mathrm{~Hz}$, and $288.6 \mathrm{~Hz}$ as shown in Figure 5. In order to further study the composition of frequency components, the inertial force and coupling force analysis are shown in Figure 6.

The mainly frequency of $24.88 \mathrm{~Hz}$ and $49.75 \mathrm{~Hz}$ caused by inertia force and coupling force can be seen from Figure 6, 

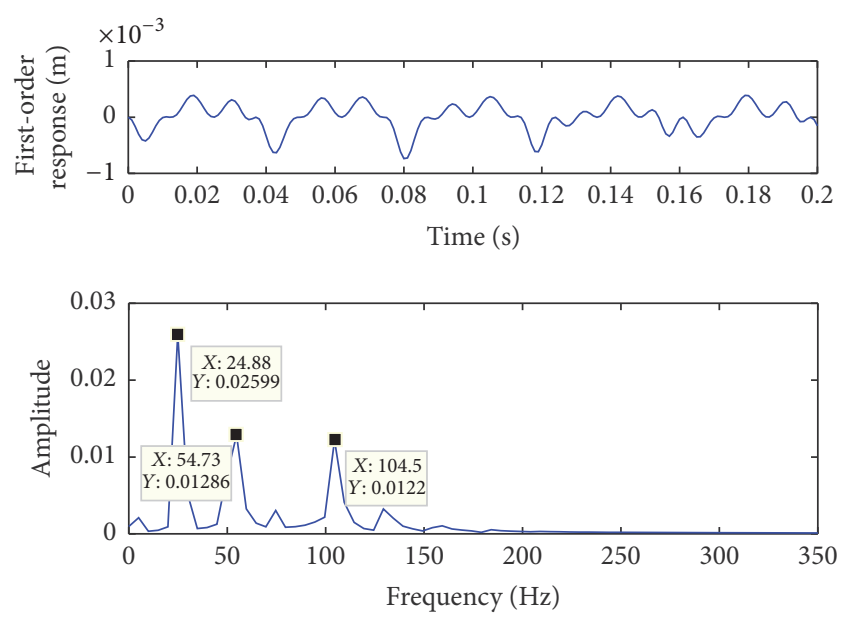

FIGURE 4: The first-order response and amplitude-frequency characteristics of the first flexible intermediate link.
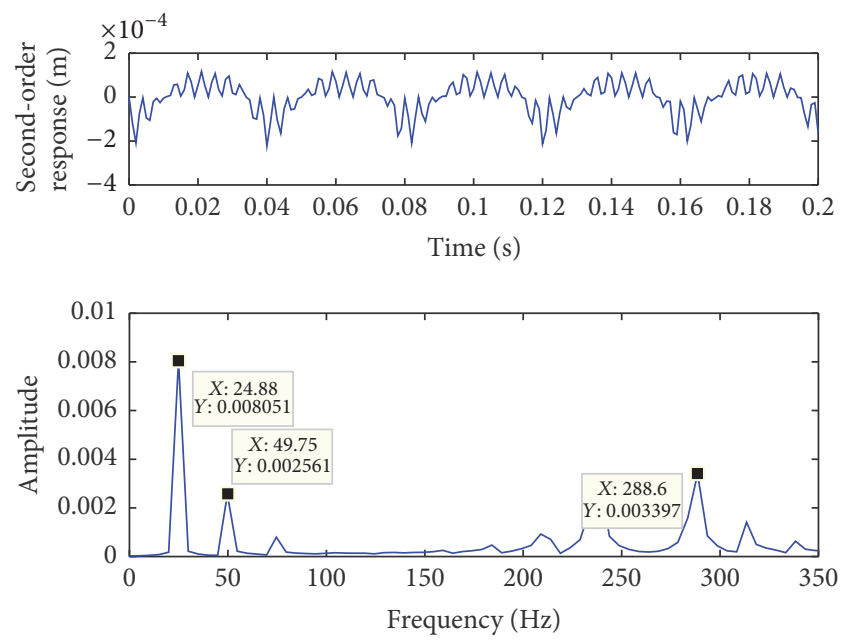

FIGURE 5: The second-order response and amplitude-frequency characteristics of the first flexible intermediate link.

which also represents that the frequency $104.5 \mathrm{~Hz}$ in Figure 4 is natural frequency and the frequency $104.5 \mathrm{~Hz}$ in Figure 5 is second-order frequency of the flexible intermediate link. The driving torque of the motor can also be obtained through the dynamic equation, which provides the basis for the motor selection.

It can be seen from Figure 7 that the maximum driving torque of the motor does not exceed $20 \mathrm{~N} \cdot \mathrm{m}$.

\section{Modal Test Verification}

In order to verify the correctness of the dynamic model established in this paper, the modal test bench is built as shown in Figure 8. The test bench is mainly composed of computer, DH5927N dynamic tester, hammer, piezoelectric acceleration sensor, charge adapter, and flexible 3-RRR FPM. A total of 11 test points were set up, among which the measuring points 1 and 11 were at the bearing, so no measurement was made.
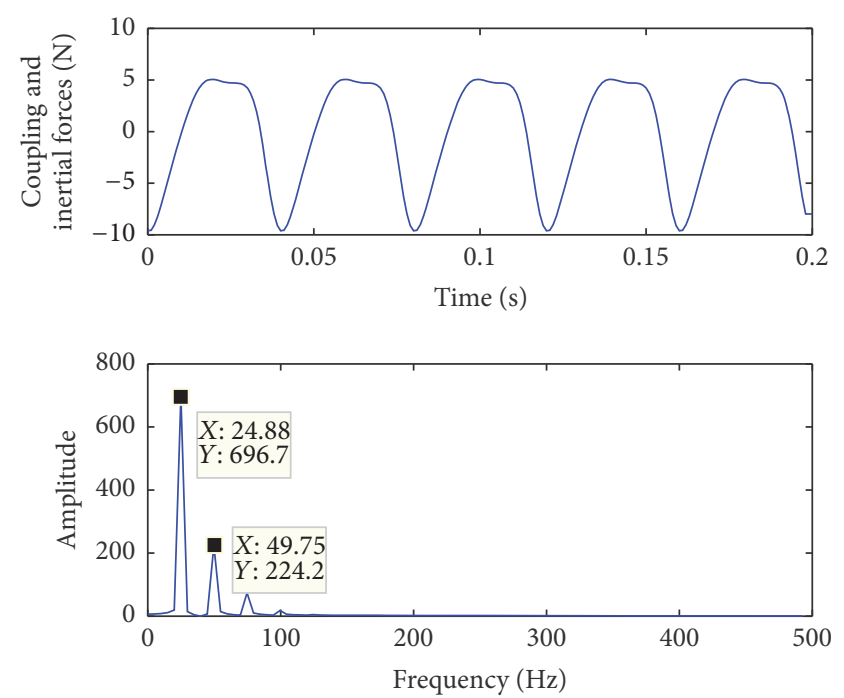

FIGURE 6: The response and amplitude-frequency characteristics of inertial force and coupling force.

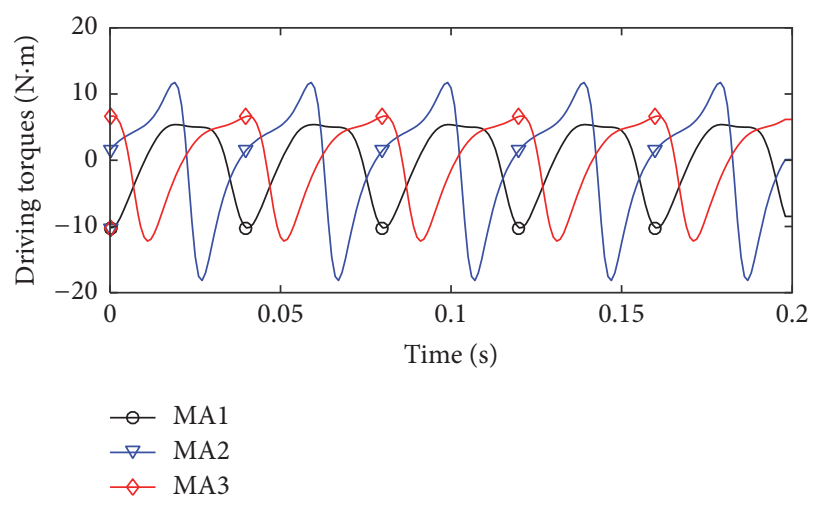

FIGURE 7: Three motor drive torque.

During the course of the experiment, the three drive motors are locked in the initial position and the end moving platform is in the central position. The multipoint excitation and the pick-up one point test method is implemented. In order to achieve a more accurate result, the test is performed 5 times repeatedly, and then the results are averaged. The test data is collected and recorded by the DH5927N dynamic tester. The dynamic response is analyzed by PolyLscf method, and the results are shown in Figure 9.

The results show that the first-order frequency is mainly around $103.1 \mathrm{~Hz}$ and the damping ratio is 0.056 . In addition the second-order vibration frequency is around $286.3 \mathrm{~Hz}$ and the damping ratio is 0.032 . Compared with the frequency characteristics obtained through modal experiment and theoretical calculation, the first-order frequency $103.1 \mathrm{~Hz}$ and the second-order frequency $286.3 \mathrm{~Hz}$ obtained through modal test are basically consistent with the theoretical results $104.5 \mathrm{~Hz}$ and $288.6 \mathrm{~Hz}$, which proves the correctness of the dynamic model established using the extended Hamilton principle and the AMM with the pinned-free boundary condition. Compared with the results obtained by 


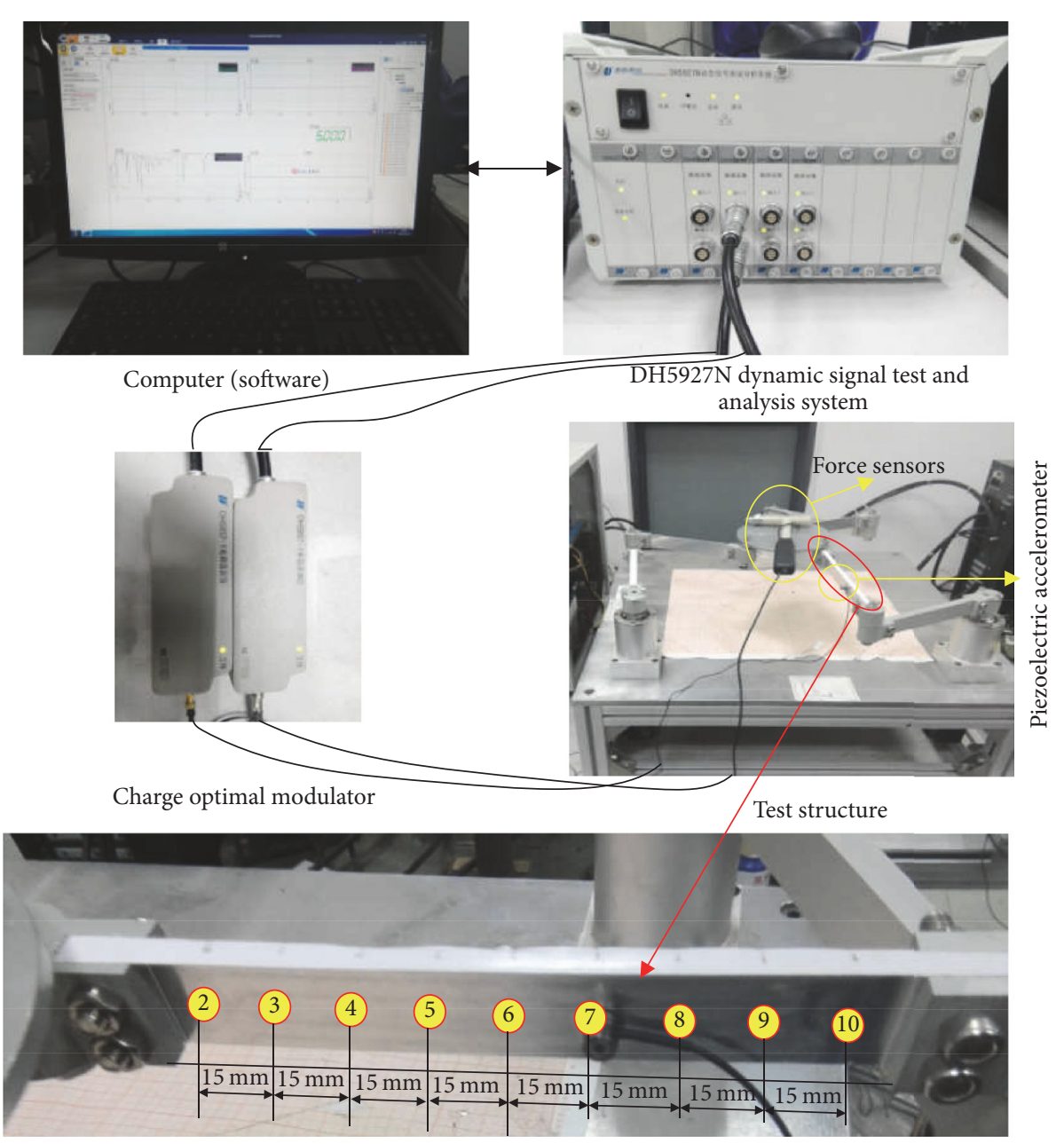

FIgURE 8: Modal test bench.

TABLE 1: The results comparison.

\begin{tabular}{lcc}
\hline Project & The results of this article & The results of others \\
\hline First-order simulation frequency $\left(S_{1}\right)$ & $103.1 \mathrm{~Hz}$ & $70.5 \mathrm{~Hz}$ \\
First-order experiment frequency $\left(E_{1}\right)$ & $104.5 \mathrm{~Hz}$ & $76.6 \mathrm{~Hz}$ \\
Second-order simulation frequency $\left(S_{2}\right)$ & $286.3 \mathrm{~Hz}$ & $280.8 \mathrm{~Hz}$ \\
Second-order experiment frequency $\left(E_{2}\right)$ & $288.6 \mathrm{~Hz}$ & $231.2 \mathrm{~Hz}$ \\
First-order relative error $\left(S_{1}-E_{1}\right) / S_{1} \times 100 \%$ & $1.36 \%$ & $8.65 \%$ \\
Second-order relative error $\left(S_{2}-E_{2}\right) / S_{2} \times 100 \%$ & $0.8 \%$ & $17.66 \%$ \\
\hline
\end{tabular}

other researchers [20], the model established in this paper has higher accuracy. The results' comparison is shown in Table 1.

The source of error between present work and others in the results is mainly in the selection of boundary conditions. This paper chooses the pinned-free boundary condition for the flexible intermediate link in the establishment of the mathematical model, while the boundary condition in the literature [20] is the pinned-pinned, and the joint gap caused by the machining and installation accuracy in the actual test bench is also part of the source of the error.

\section{Conclusions}

In this paper, based on the extended Hamilton principle and the AMM, the coupled dynamic model of planar 3-RRR FPM with high precision and less dynamic parameters is established with pinned-free boundary condition. This model 


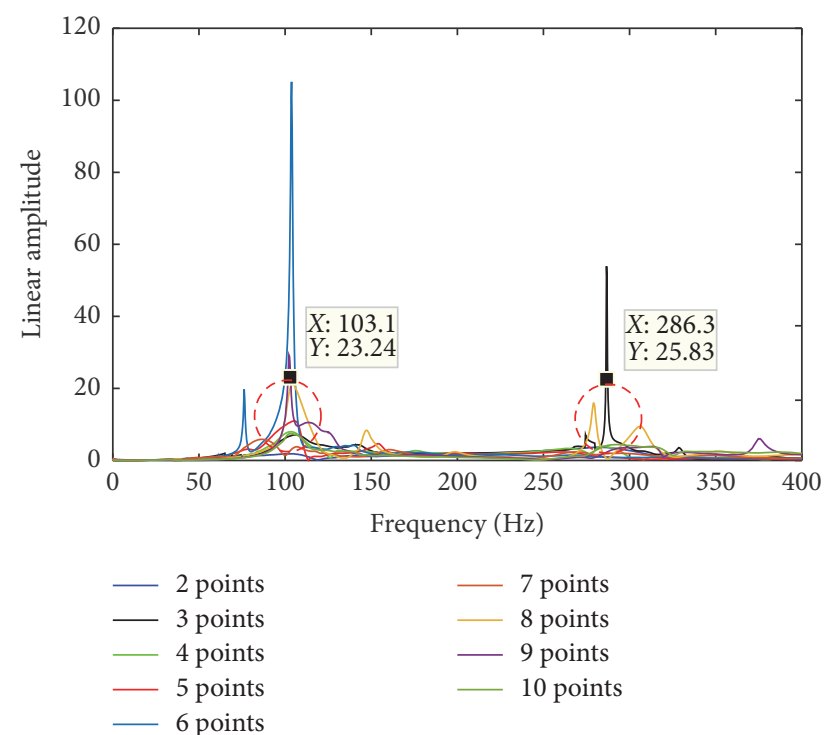

FIGURE 9: Modal test results. takes into account the concentrated moment of inertia and rigid-flexible coupling effect on system dynamic. The firstorder frequency $103.1 \mathrm{~Hz}$ and the second-order frequency $286.3 \mathrm{~Hz}$ obtained through modal experiment are very close to the theoretical results $104.5 \mathrm{~Hz}$ and $288.6 \mathrm{~Hz}$. The research indicates that the established dynamic model has a high accuracy. Based on the established dynamic model, it is found that the inertial force and the coupling force can cause the forced vibration, the vibration frequencies are $24.88 \mathrm{~Hz}$ and $49.75 \mathrm{~Hz}$, and the driving torque of the motor does not exceed $20 \mathrm{~N} \cdot \mathrm{m}$ which can provide a basis for motor selection. The rigid-flexible coupling dynamics modeling method proposed in this paper is applicable to all kinds of FPM with flexible link. The model has high precision and high efficiency, which solves the problem of complexity of dynamics model and is of great significance to the controller design based on the dynamic model.

\section{Appendix}

$$
\begin{aligned}
& M=\left[\begin{array}{cccc}
M_{11} & M_{12} & 0 & M_{14} \\
M_{21} & M_{22} & 0 & M_{24} \\
0 & 0 & M_{33} & 0 \\
M_{41} & M_{42} & 0 & M_{44}
\end{array}\right] \in R^{(9+3 r) \times(9+3 r)}, \\
& M_{11}=\left[\begin{array}{ccc}
J_{A_{1}}+m_{B_{1}} L_{1}^{2}+\frac{4 m_{1} L_{1}^{2}}{3} & 0 & 0 \\
0 & J_{A_{2}}+m_{B_{2}} L_{2}^{2}+\frac{4 m_{2} L_{2}^{2}}{3} & 0 \\
0 & 0 & J_{A_{3}}+m_{B_{3}} L_{3}^{2}+\frac{4 m_{3} L_{3}^{2}}{3}
\end{array}\right] \in R^{3 \times 3},
\end{aligned}
$$$$
M_{12}=\left[\begin{array}{ccc}
\frac{m_{1} C_{1} L_{1}^{2}}{2}+\int_{0}^{L_{1}} \rho_{A_{1}} S_{1} L_{1} \sum_{j=1}^{r} \varphi_{1 j} q_{1 j} d x & 0 & 0 \\
0 & \frac{m_{2} C_{2} L_{2}^{2}}{2}+\int_{0}^{L_{2}} \rho_{A_{2}} S_{2} L_{2} \sum_{j=1}^{r} \varphi_{2 j} q_{2 j} d x & 0 \\
0 & 0 & \frac{m_{3} C_{3} L_{3}^{2}}{2}+\int_{0}^{L_{3}} \rho_{A_{3}} S_{3} L_{3} \sum_{j=1}^{r} \varphi_{3 j} q_{3 j} d x
\end{array}\right] \in R^{3 \times 3},
$$$$
M_{14}=\left[\begin{array}{ccc}
\int_{0}^{L_{1}} \rho_{A_{1}} C_{1} L_{1} \varphi_{11} d x \cdots \int_{0}^{L_{1}} \rho_{A_{1}} C_{1} L_{1} \varphi_{1 r} d x & 0 \cdots 0 & 0 \cdots 0 \\
0 \cdots 0 & \int_{0}^{L_{2}} \rho_{A_{2}} C_{2} L_{2} \varphi_{21} d x \cdots \int_{0}^{L_{2}} \rho_{A_{2}} C_{2} L_{2} \varphi_{2 r} d x & 0 \cdots 0 \\
0 \cdots 0 & 0 \cdots 0 & \int_{0}^{L_{3}} \rho_{A_{3}} C_{3} L_{3} \varphi_{31} d x \cdots \int_{0}^{L_{3}} \rho_{A_{3}} C_{3} L_{3} \varphi_{3 r} d x
\end{array}\right]
$$

$$
\in R^{3 \times 3 r}
$$

$M_{21}$

$$
=\left[\begin{array}{ccc}
\frac{m_{1} C_{1} L_{1}^{2}}{2}-\frac{m_{1} S_{1} L_{1}^{2}}{2}+\int_{0}^{L_{1}} \rho_{A_{1}} S_{1} L_{1} \sum_{j=1}^{r} \varphi_{1 j} q_{1 j} d x & 0 & 0 \\
0 & \frac{m_{2} C_{2} L_{2}^{2}}{2}-\frac{m_{2} S_{2} L_{2}^{2}}{2}+\int_{0}^{L_{2}} \rho_{A_{2}} S_{2} \sum_{j=1}^{r} \varphi_{2 j} q_{2 j} d x & 0 \\
0 & 0 & \frac{m_{3} C_{3} L_{3}^{2}}{2}-\frac{m_{3} S_{3} L_{3}^{2}}{2}+\int_{0}^{L_{3}} \rho_{A_{3}} \varphi_{3} \sum_{j=1}^{r} Y_{3 j} q_{3 j} d x
\end{array}\right]
$$

$\in R^{3 \times 3}$ 
Shock and Vibration

9

$$
\begin{aligned}
& M_{22}=\left[\begin{array}{ccc}
J_{B_{1}}+\frac{m_{1} L_{1}^{2}}{3}+\int_{0}^{L_{1}} \rho_{A_{1}}\left(\sum_{j=1}^{r} \varphi_{1 j} q_{1 j}\right)^{2} d x & 0 & 0 \\
0 & J_{B_{2}}+\frac{m_{2} L_{2}^{2}}{3}+\int_{0}^{L_{2}} \rho_{A_{2}}\left(\sum_{j=1}^{r} \varphi_{2 j} q_{2 j}\right)^{2} d x & 0 \\
0 & 0 & J_{B_{3}}+\frac{m_{3} L_{3}^{2}}{3}+\int_{0}^{L_{3}} \rho_{A_{3}}\left(\sum_{j=1}^{r} \varphi_{1 j} q_{1 j}\right)^{2} d x
\end{array}\right] \in R^{3 \times 3}, \\
& M_{24}=\left[\begin{array}{ccc}
\int_{0}^{L_{1}} \rho_{A_{1}} x \varphi_{11} d x \cdots \int_{0}^{L_{1}} \rho_{A_{1}} x \varphi_{1 r} d x & 0 \cdots 0 & 0 \cdots 0 \\
0 \cdots 0 & \int_{0}^{L_{2}} \rho_{A_{2}} x \varphi_{21} d x \cdots \int_{0}^{L_{2}} \rho_{A_{2}} x \varphi_{2 r} d x & 0 \cdots 0 \\
0 \cdots 0 & 0 \cdots 0 & \int_{0}^{L_{3}} \rho_{A_{3}} x \varphi_{31} d x \cdots \int_{0}^{L_{3}} \rho_{A_{3}} x \varphi_{3 r} d x
\end{array}\right] \in R^{3 \times 3 r}, \\
& M_{33}=\left[\begin{array}{ccc}
m_{p} & 0 & 0 \\
0 & m_{p} & 0 \\
0 & 0 & J_{\varphi p}
\end{array}\right] \in R^{3 \times 3}, \\
& M_{41}=\left[\begin{array}{ccc}
-\int_{0}^{L_{1}} \rho_{A_{1}} C_{1} L_{1} \varphi_{11} d x & 0 & 0 \\
\vdots & \vdots & \vdots \\
-\int_{0}^{L_{1}} \rho_{A_{1}} C_{1} L_{1} \varphi_{1 r} d x & 0 & 0 \\
0 & -\int_{0}^{L_{2}} \rho_{A_{2}} C_{2} L_{2} \varphi_{21} d x & 0 \\
\vdots & \vdots & \vdots \\
0 & -\int_{0}^{L_{2}} \rho_{A_{2}} C_{2} L_{2} \varphi_{2 r} d x & 0 \\
0 & 0 & -\int_{0}^{L_{3}} \rho_{A_{3}} C_{3} L_{3} \varphi_{31} d x \\
\vdots & \vdots & \vdots \\
0 & 0 & -\int_{0}^{L_{3}} \rho_{A_{3}} C_{3} L_{3} \varphi_{3 r} d x
\end{array}\right] \\
& M_{42}=\left[\begin{array}{ccc}
\int_{0}^{L_{1}} \rho_{A_{1}} x \varphi_{11}(x) d x+J_{B_{1}} \varphi_{11}^{\prime 2}(0) & 0 & 0 \\
\vdots & \vdots & \vdots \\
\int_{0}^{L_{1}} \rho_{A_{1}} x \varphi_{1 r}(x) d x+J_{B_{1}} \varphi_{1 r}^{\prime 2}(0) & 0 & 0 \\
0 & \int_{0}^{L_{2}} \rho_{A_{2}} x \varphi_{21}(x) d x+J_{B_{2}} \varphi_{21}^{\prime 2}(0) & 0 \\
\vdots & \vdots & \vdots \\
0 & \int_{0}^{L_{2}} \rho_{A_{2}} x \varphi_{2 r}(x) d x+J_{B_{2}} \varphi_{2 r}^{\prime 2}(0) & 0 \\
0 & 0 & \int_{0}^{L_{3}} \rho_{A_{3}} x \varphi_{31}(x) d x+J_{B_{3}} \varphi_{31}^{\prime 2}(0) \\
0 & \vdots & \vdots \\
0 & 0 & \int_{0}^{L_{3}} \rho_{A_{3}} x \varphi_{3 r}(x) d x+J_{B_{3}} \varphi_{3 r}^{\prime 2}(0)
\end{array}\right],
\end{aligned}
$$


10

Shock and Vibration

$$
\begin{aligned}
& M_{44}=\left[\begin{array}{ccc}
M_{44}^{1} & 0 & 0 \\
0 & M_{44}^{2} & 0 \\
0 & 0 & M_{44}^{3}
\end{array}\right] \in R^{3 r \times 3 r}, \\
& M_{44}^{i}=\left[\begin{array}{ccc}
\int_{0}^{L_{i}} \rho_{A_{i}} \varphi_{i 1}^{2}(x) d x+J_{B_{1}} \varphi_{i 1}^{\prime 2}(0) & \cdots & 0 \\
\vdots & \cdots & \vdots \\
0 & \cdots & \int_{0}^{L_{i}} \rho_{A_{i}} \varphi_{i r}^{2}(x) d x+J_{B_{i}} \varphi_{i r}^{2}(0)
\end{array}\right] \in R^{r \times r} \\
& C=\left[\begin{array}{cccc}
0 & C_{12} & 0 & C_{14} \\
C_{21} & C_{22} & 0 & C_{24} \\
0 & 0 & 0 & 0 \\
C_{41} & C_{42} & 0 & 0
\end{array}\right] \in R^{(9+3 r) \times(9+3 r)}, \\
& C_{12}=\left[\begin{array}{ccc}
C_{12}^{1} & 0 & 0 \\
0 & C_{12}^{2} & 0 \\
0 & 0 & C_{12}^{3}
\end{array}\right] \in R^{3 \times 3}, \\
& C_{12}^{i}=\frac{m_{i} S_{i} \dot{\beta}_{i} L_{i}^{2}}{2}+\int_{0}^{L_{i}} \rho_{A_{i}} S_{i} L_{i} \sum_{j=1}^{r} \varphi_{i j} \dot{q}_{i j} d x-\int_{0}^{L_{i}} \rho_{A_{i}} C_{i} \dot{\beta}_{i} L_{i} \sum_{j=1}^{r} \varphi_{i j} q_{i j} d x, \\
& C_{14} \\
& =\left[\begin{array}{ccc}
\int_{0}^{L_{1}} \rho_{A_{1}} S_{1} \dot{\beta}_{1} L_{1} \varphi_{11} d x \cdots \int_{0}^{L_{1}} \rho_{A_{1}} S_{1} \dot{\beta}_{1} L_{1} \varphi_{1 r} d x & 0 \cdots 0 & 0 \cdots 0 \\
0 \cdots 0 & \int_{0}^{L_{2}} \rho_{A_{2}} S_{2} \dot{\beta}_{2} L_{2} \varphi_{21} d x \cdots \int_{0}^{L_{2}} \rho_{A_{2}} S_{2} \dot{\beta}_{2} L_{2} \varphi_{2 r} d x & 0 \cdots 0 \\
0 \cdots 0 & 0 \cdots 0 & \int_{0}^{L_{3}} \rho_{A_{3}} S_{3} \dot{\beta}_{3} L_{3} \varphi_{31} d x \cdots \int_{0}^{L_{3}} \rho_{A_{3}} S_{3} \dot{\beta}_{3} L_{3} \varphi_{3 r} d x
\end{array}\right] \\
& \in R^{3 \times 3 r} \\
& C_{21}=\left[\begin{array}{ccc}
\int_{0}^{L_{1}} \rho_{A_{1}} C_{1} L_{1} \dot{\alpha}_{1} \sum_{j=1}^{r} \varphi_{1 j} q_{1 j} d x & 0 & 0 \\
0 & \int_{0}^{L_{2}} \rho_{A_{2}} C_{2} L_{2} \dot{\alpha}_{2} \sum_{j=1}^{r} \varphi_{2 j} q_{2 j} d x & 0 \\
0 & 0 & \int_{0}^{L_{3}} \rho_{A_{3}} C_{3} L_{3} \dot{\alpha}_{3} \sum_{j=1}^{r} \varphi_{3 j} q_{3 j} d x
\end{array}\right] \in R^{3 \times 3} \\
& C_{22}=\left[\begin{array}{ccc}
\int_{0}^{L_{1}} \rho_{A_{1}}\left(\sum_{j=1}^{r} \varphi_{1 j} q_{1 j}\right)\left(\sum_{j=1}^{r} \varphi_{1 j} \dot{q}_{1 j}\right) d x & 0 \\
0 & \int_{0}^{L_{2}} \rho_{A_{2}}\left(\sum_{j=1}^{r} \varphi_{2 j} q_{2 j}\right)\left(\sum_{j=1}^{r} \varphi_{2 j} \dot{q}_{2 j}\right) d x & 0 \\
0 & 0 & \int_{0}^{L_{3}} \rho_{A_{3}}\left(\sum_{j=1}^{r} \varphi_{3 j} q_{3 j}\right)\left(\sum_{j=1}^{r} \varphi_{3 j} \dot{q}_{3 j}\right) d x
\end{array}\right] \in R^{3 \times 3}, \\
& C_{24}=\left[\begin{array}{ccc}
\int_{0}^{L_{1}} \rho_{A_{1}} \dot{\beta}_{1} \varphi_{11}^{2} q_{11} d x \cdots \int_{0}^{L_{1}} \rho_{A_{1}} \dot{\beta}_{1} \varphi_{1 r}^{2} q_{1 r} d x & 0 \cdots 0 & 0 \cdots 0 \\
0 \cdots 0 & \int_{0}^{L_{2}} \rho_{A_{2}} \dot{\beta}_{2} \varphi_{21}^{2} q_{21} d x \cdots \int_{0}^{L_{2}} \rho_{A_{2}} \dot{\beta}_{2} \varphi_{21}^{2} q_{21} d x & 0 \cdots 0 \\
0 \cdots 0 & 0 \cdots 0 & \int_{0}^{L_{3}} \rho_{A_{3}} \dot{\beta}_{3} \varphi_{31}^{2} q_{31} d x \cdots \int_{0}^{L_{3}} \rho_{A_{3}} \dot{\beta} \varphi_{31}^{2} q_{31} d x
\end{array}\right]
\end{aligned}
$$


Shock and Vibration

11

$$
\begin{aligned}
& \in R^{3 \times 3 r}
\end{aligned}
$$

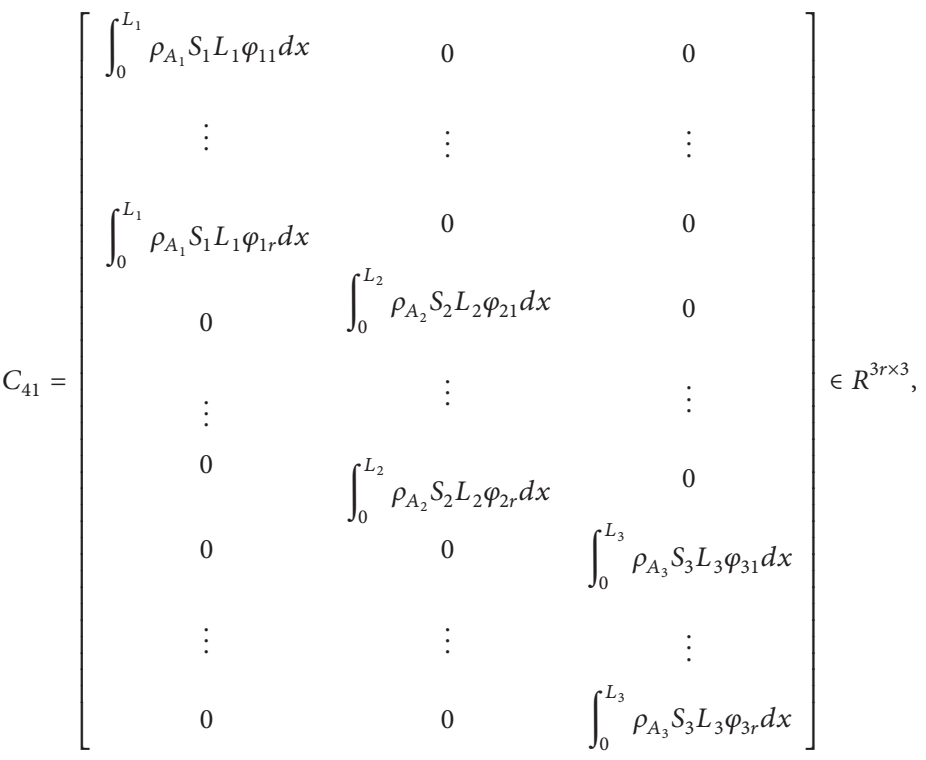

$$
\begin{aligned}
& C_{42}=\left[\begin{array}{ccc}
\int_{0}^{L_{1}} \rho_{A_{1}} \dot{\beta}_{1} \varphi_{11}^{2} q_{11} d x & 0 & 0 \\
\vdots & \vdots & \vdots \\
\int_{0}^{L_{1}} \rho_{A_{1}} \dot{\beta}_{1} \varphi_{1 r}^{2} q_{1 r} d x & 0 & 0 \\
0 & \int_{0}^{L_{2}} \rho_{A_{2}} \dot{\beta}_{2} \varphi_{21}^{2} q_{21} d x & 0 \\
\vdots & \vdots & \vdots \\
0 & \int_{0}^{L_{2}} \rho_{A_{2}} \dot{\beta}_{2} \varphi_{2 r}^{2} q_{2 r} d x & 0 \\
0 & 0 & \int_{0}^{L_{3}} \rho_{A_{3}} \dot{\beta}_{3} \varphi_{31}^{2} q_{31} d x \\
\vdots & \vdots & \vdots \\
0 & 0 & \int_{0}^{L_{3}} \rho_{A_{3}} \dot{\beta}_{3} \varphi_{3 r}^{2} q_{3 r} d x
\end{array}\right] \in R^{3 \times 3}, \\
& K=\left[\begin{array}{cccc}
0 & 0 & 0 & 0 \\
0 & 0 & 0 & 0 \\
0 & 0 & 0 & 0 \\
0 & 0 & 0 & K_{f}
\end{array}\right] \in R^{(9+3 r) \times(9+3 r)}, \\
& K_{f}=\left[\begin{array}{ccc}
K_{f}^{1} & 0 & 0 \\
0 & K_{f}^{2} & 0 \\
0 & 0 & K_{f}^{3}
\end{array}\right] \in R^{3 r \times 3 r},
\end{aligned}
$$

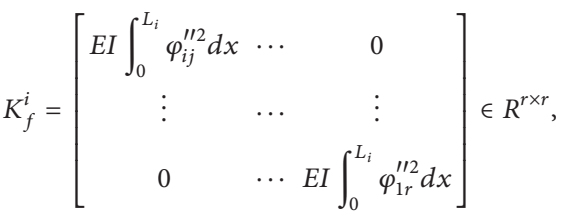

$$
\begin{aligned}
& J_{f}=\left[J_{f \alpha} ; J_{f \beta} ; J_{f p} ; J_{f w}\right] \in R^{(3 r+9) \times 6} \text {, } \\
& J_{f \alpha}=\left[\begin{array}{cccccc}
-a_{1 y} & a_{1 x} & 0 & 0 & 0 & 0 \\
0 & 0 & -a_{2 y} & a_{2 x} & 0 & 0 \\
0 & 0 & 0 & 0 & -a_{3 y} & a_{3 x}
\end{array}\right] \in R^{3 \times 6}, \\
& \bar{a}_{i}=\left(a_{1 x}, a_{1 y}\right)=\left(L_{i} \cos \alpha_{i}, L_{i} \sin \alpha_{i}\right),
\end{aligned}
$$




$$
\begin{aligned}
& J_{f \beta}=\left[\begin{array}{cccccc}
-b_{1 y} & b_{1 x} & 0 & 0 & 0 & 0 \\
0 & 0 & -b_{2 y} & b_{2 x} & 0 & 0 \\
0 & 0 & 0 & 0 & -b_{3 y} & b_{3 x}
\end{array}\right] \in R^{3 \times 6}, \\
& \bar{b}_{i}=\left(b_{1 x}, b_{1 y}\right)=\left(L_{i} \cos \beta_{i}-\sum_{j=1}^{r} \varphi_{i j}\left(L_{i}\right) q_{i j} \sin \beta_{i}, L_{i} \sin \beta_{i}+\sum_{j=1}^{r} \varphi_{i j}\left(L_{i}\right) q_{i j} \cos \beta_{i}\right), \\
& J_{f w}=\left[\begin{array}{ccc}
J_{f w}^{1} & 0 & 0 \\
0 & J_{f w}^{2} & 0 \\
0 & 0 & J_{f w}^{3}
\end{array}\right] \in R^{3 r \times 6} .
\end{aligned}
$$

\section{Nomenclature}

$A_{i}:$
$B_{i}, C_{i}:$
$L_{i}:$
$R:$
$\alpha_{i}:$
$\beta_{i}:$
$x_{i}, y_{i}:$
$x_{a i}: y_{a i}:$
$T_{i}:$
$T_{A_{i}}:$
$T_{A_{i} B_{i}}:$
$T_{B_{i}}:$
$T_{B_{i} C_{i}}:$
$w_{i}\left(x_{i}, t\right)$
$r_{A_{i}}:$
$\rho_{B_{i}}:$
$\rho_{A_{i}}:$
$\vec{k}_{i}:$
$m_{B_{i}}:$

$A_{i}$ :

$B_{i}, C_{i}$ :

$L_{i}$ :

$\alpha_{i}:$

$\beta_{i}: \quad$ Angle between the flexible intermediate

Distance between a point and joint $A_{i}$ on

driving link in the $x, y$ direction

Passive revolute joint

Rod length

Radius of the circle of joints in moving platform

Angle between the driving link and the

Distance between a point and joint $B_{i}$ on

flexible intermediate link in the $x, y$

direction

Total kinetic energy of the $i$ th branch

Rotational kinetic energy of the $i$ th drive joint and the motor rotor

Kinetic energy of the $i$ th driving rod

Kinetic energy of the joint $B_{i}$

Kinetic energy of the $i$ th flexible

intermediate link

Transverse elastic displacement of $x$ points

on flexible intermediate link

Assumed mode

Material density

Unit length mass of the $i$ th flexible

intermediate link

Mass of the joint $B_{i}$

Rotational inertia of the driving joint $A_{i}$

and the motor rotor

Rotational inertia of joint $B_{i}$

Vector coordinates of the $i$ th drive rod

Vector coordinates of the $i$ th flexible

intermediate link

$\left(\alpha_{i}, \beta_{i}, \dot{w}\right)\left(w^{\prime}\right)$ : Differential for the time and displace-

ment, respectively

$V_{i}: \quad$ Potential energy of the $i$ th branch

$E_{i}: \quad \quad$ Elastic modulus

$I\left(x_{i}\right)$ :

Moment of inertia of section of flexible

intermediate link

$\delta W_{i}:$

Virtual work of the $i$ th branch

$\bar{f}_{i x y}$ :

\begin{tabular}{|c|c|}
\hline$M_{A_{i}}:$ & $\begin{array}{l}\text { Drive torque of the } \\
i \text { th servomotor }\end{array}$ \\
\hline$\delta \alpha_{i}, \delta \beta_{i}, \delta w_{i}, \delta w_{B_{i}}, \delta w_{B_{i}}^{\prime}, \delta w_{C_{i}}, \delta w_{C_{i}}^{\prime}:$ & $\begin{array}{l}\text { Virtual angle } \\
\text { displacement }\end{array}$ \\
\hline $\bar{\eta}_{i}:$ & $\begin{array}{l}\text { Generalized variable } \\
\text { of the } i \text { th branch }\end{array}$ \\
\hline$q_{i j}(t):$ & $\begin{array}{l}\text { Unknown } \\
\text { generalized elastic } \\
\text { variable of the } i \text { th } \\
\text { flexible intermediate } \\
\text { link }\end{array}$ \\
\hline$\varphi_{i j}\left(x_{i}\right):$ & $\begin{array}{l}\text { Mode function } \\
\text { corresponding to the } \\
\text { known boundary } \\
\text { condition }\end{array}$ \\
\hline$m_{p}:$ & $\begin{array}{l}\text { Quality of the } \\
\text { moving platform }\end{array}$ \\
\hline$J_{\varphi p}:$ & $\begin{array}{l}\text { Moment of inertia of } \\
\text { the moving platform }\end{array}$ \\
\hline$\left(x_{c i}^{\prime}, y_{c i}^{\prime}\right):$ & $\begin{array}{l}\text { Coordinate of the } \\
\text { joint } C_{i} \text { in the local } \\
\text { coordinate system }\end{array}$ \\
\hline$M:$ & $\begin{array}{l}\text { Positive definite } \\
\text { symmetric mass } \\
\text { matrix }\end{array}$ \\
\hline$C:$ & $\begin{array}{l}\text { Centrifugal force } \\
\text { and Coriolis matrix }\end{array}$ \\
\hline$K:$ & Stiffness matrix \\
\hline$x_{p} y_{p} \varphi_{p}:$ & $\begin{array}{l}\text { Motion coordinate } \\
\text { of moving platform }\end{array}$ \\
\hline $\bar{\eta}:$ & $\begin{array}{l}\text { Generalized } \\
\text { coordinate variable }\end{array}$ \\
\hline $\bar{\alpha}, \bar{\beta}, \bar{X}_{P}:$ & $\begin{array}{l}\text { Rigid body motion } \\
\text { coordinate }\end{array}$ \\
\hline $\bar{q}:$ & $\begin{array}{l}\text { Elastic coordinate of } \\
\text { three flexible } \\
\text { intermediate links }\end{array}$ \\
\hline $\bar{P}$ & $\begin{array}{l}\text { Driving torque of } \\
\text { the three motors }\end{array}$ \\
\hline$J_{f}:$ & Binding matrix \\
\hline $\bar{f}:$ & $\begin{array}{l}\text { Constraints of } \\
\text { internal force of the } \\
\text { joint } C_{i}\end{array}$ \\
\hline$i=1,2,3:$ & $\begin{array}{l}\text { The } 1,2,3 \text { branch, } \\
\text { respectively. }\end{array}$ \\
\hline
\end{tabular}

Constraint force between the joint $C_{i}$ and the end of the flexible intermediate link 


\section{Conflicts of Interest}

The authors declare that there are no conflicts of interest regarding the publication of this paper.

\section{Acknowledgments}

This research work was supported by the National Natural Science Foundation of China (no. 51305444, no. 51307172, and no. U1610111), the Scientific and Technological Project of Jiangsu Province (BY201402806), the Project Funded by the Priority Academic Program Development of Jiangsu Higher Education Institutions (PAPD), and Six Talent Peaks Project in Jiangsu Province (ZBZZ-041).

\section{References}

[1] B. Li, "Dynamic analysis of a 3-DOF parallel manipulator," Journal of Northeastern University (Natural Science), vol. 31, no. 11, pp. 1608-1610, 2010.

[2] S. K. Dwivedy and P. Eberhard, "Dynamic analysis of flexible manipulators, a literature review," Mechanism and Machine Theory, vol. 41, no. 7, pp. 749-777, 2006.

[3] J. Chen, P. C. Hagan, and S. Saydam, "Parametric study on the axial performance of a fully grouted cable bolt with a new pullout test," International Journal of Mining Science and Technology, vol. 26, no. 1, pp. 53-58, 2016.

[4] K. M. Zheng and Q. J. Zhang, "Elastic dynamics and analysis of vibration characteristics of delta robot with joint clearance," Transactions of the Chinese Society of Agricultural Engineering, vol. 31, no. 14, pp. 39-48, 2015.

[5] J. Song, T. Zhang, L. Xu, and X. Tang, "Research actuality and prospect of picking robot for fruits and vegetables," Transactions of the Chinese Society of Agricultural Machinery, vol. 37, no. 5, pp. 158-162, 2006.

[6] Q. C. Li, T. Hu, and C. Y. Wu, "Review of end-effectors in fruit and vegetable harvesting robot," Transactions of the Chinese Society for Agricultural Machinery, vol. 39, no. 3, pp. 175-179, 2008.

[7] S. Arabzadeh Jarkani, H. Khoshdast, E. Shariat, and A. Sam, "Modeling the effects of mechanical parameters on the hydrodynamic behavior of vertical current classifiers," International Journal of Mining Science and Technology, vol. 24, no. 1, pp. 123127, 2014.

[8] X. Wang and J. K. Mills, "Dynamic modeling of a flexiblelink planar parallel platform using a substructuring approach," Mechanism and Machine Theory, vol. 41, no. 6, pp. 671-687, 2006.

[9] M. Loudini, D. Boukhetala, and M. Tadjine, "Comprehensive mathematical modelling of a lightweight flexible link robot manipulator," International Journal of Modelling, Identification and Control, vol. 2, no. 4, pp. 313-321, 2007.

[10] L. Meirovitch and R. Parker, "Fundamentals of vibrations," Applied Mechanics Reviews, vol. 54, no. 6, article 100, 2001.

[11] Q. Yu and J. Z. Hong, "Some topics on flexible multi-body system dynamics," Advances in Mechanics, vol. 29, no. 2, pp. 145154,2000

[12] Y. Yu, Z. Du, J. Yang, and Y. Li, "An experimental study on the dynamics of a 3-RRR flexible parallel robot," IEEE Transactions on Robotics, vol. 27, no. 5, pp. 992-997, 2011.
[13] Q. H. Zhang and X. M. Zhang, "Dynamic analysis of planar 3-RRR flexible parallel robots under uniform temperature change," Journal of Vibration and Control, vol. 21, no. 1, pp. 81104,2015

[14] Q. H. Zhang, X. R. Fang, and X. M. Zhang, "Dyanmic analysis of planar 3-RRR flexible parallel robots with dynamic stiffening," Shock and Vibration, vol. 2014, Article ID 370145, 13 pages, 2014.

[15] X. C. Zhang, X. M. Zhang, and Z. Chen, "Dynamic analysis of a 3- R RR parallel mechanism with multiple clearance joints," Mechanism \& Machine Theory, vol. 78, no. 78, pp. 105-115, 2014.

[16] A. Fattah, J. Angeles, and A. K. Misra, "Dynamics of a 3-DOF spatial parallel manipulator with flexible links," in Proceedings of the 1995 IEEE International Conference on Robotics and Automation, vol. 1, pp. 627-632, May 1995.

[17] S. J. A. Mostafavi Yazdi and S. Irani, "Transverse vibration of double cracked beam using assumed mode method," in Proceedings of the 4th International Conference on Recent Advances in Space Technologies 2009, RAST'09, pp. 156-160, June 2009.

[18] W. Chen, "Dynamic modeling of multi-link flexible robotic manipulators," Computers \& Structures, vol. 79, no. 2, pp. 183195, 2001.

[19] X. Zhang, J. K. Mills, and W. L. Cleghorn, "Dynamic modeling and experimental validation of a 3-PRR parallel manipulator with flexible intermediate links," Journal of Intelligent and Robotic Systems, vol. 50, no. 4, pp. 323-340, 2007.

[20] X. Y. Wang, "Experimental modal analysis of flexible linkages in a smart parallel platform," in Proceeding of the 7th Cansmart Meeting - International Workshop on Smart Materials and Structures, vol. 1, pp. 37-46, Montreal, Canada, 2004.

[21] B. Kang and J. K. Mills, "Dynamic modeling of structurallyflexible planar parallel manipulator," Robotica, vol. 20, no. 3, pp. 329-339, 2002.

[22] X. Zhang, J. K. Mills, and W. L. Cleghorn, "Multi-mode vibration control and position error analysis of parallel manipulator with multiple flexible links," Transactions of the Canadian Society for Mechanical Engineering, vol. 34, no. 2, pp. 197-213, 2010.

[23] L. Meirovitch, Fundamentals of Vibrations, Waveland Press, 2010.

[24] H. Y. Hu, Mechanical Vibration Foundation, Beijing University of Aeronautics and Astronautics Press, Beijing, China, 2005.

[25] S. Rao Singiresu, Mechanical Vibration, Tsinghua University Press, Beijing, China, 4th edition, Compiled by X. Y. Li and M. L. Zhang, 2009. 


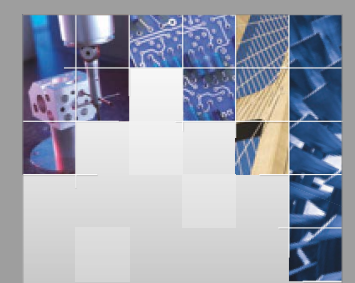

\section{Enfincering}
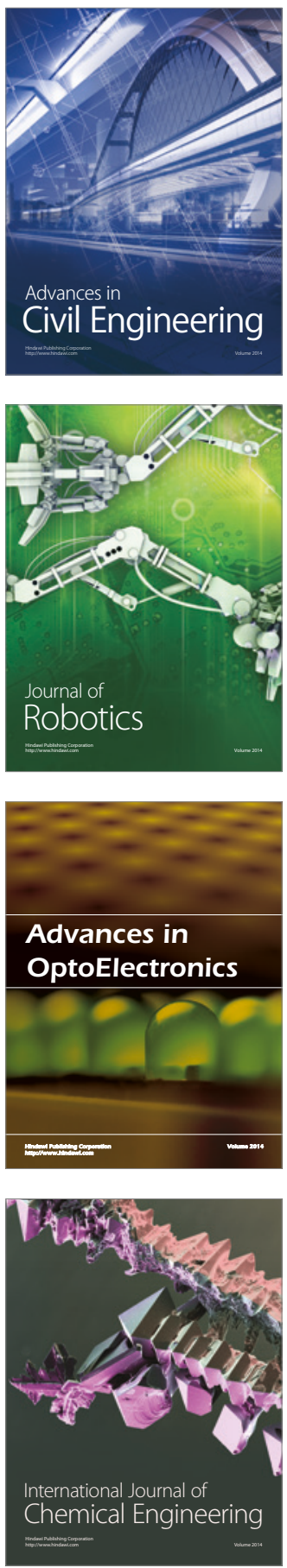

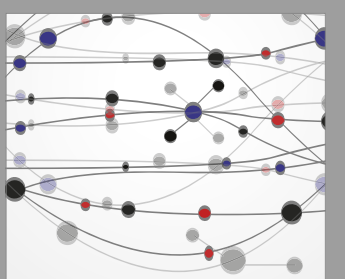

The Scientific World Journal

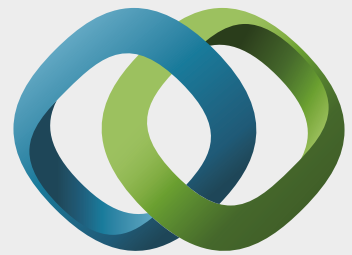

\section{Hindawi}

Submit your manuscripts at

https://www.hindawi.com
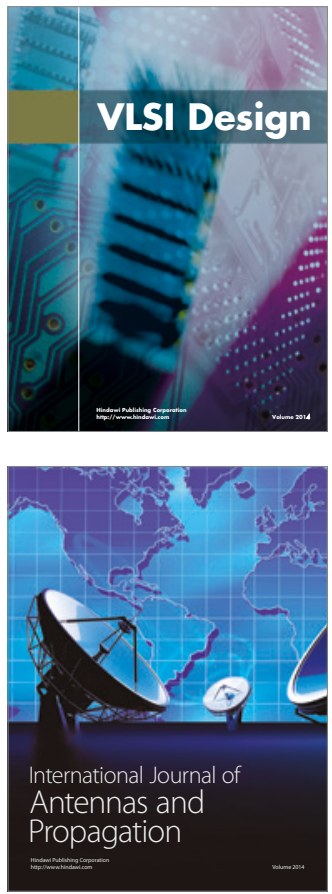

\section{Rotating}

Machinery
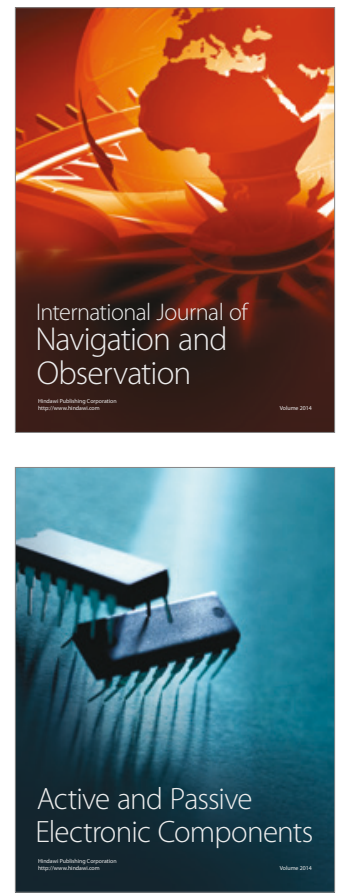
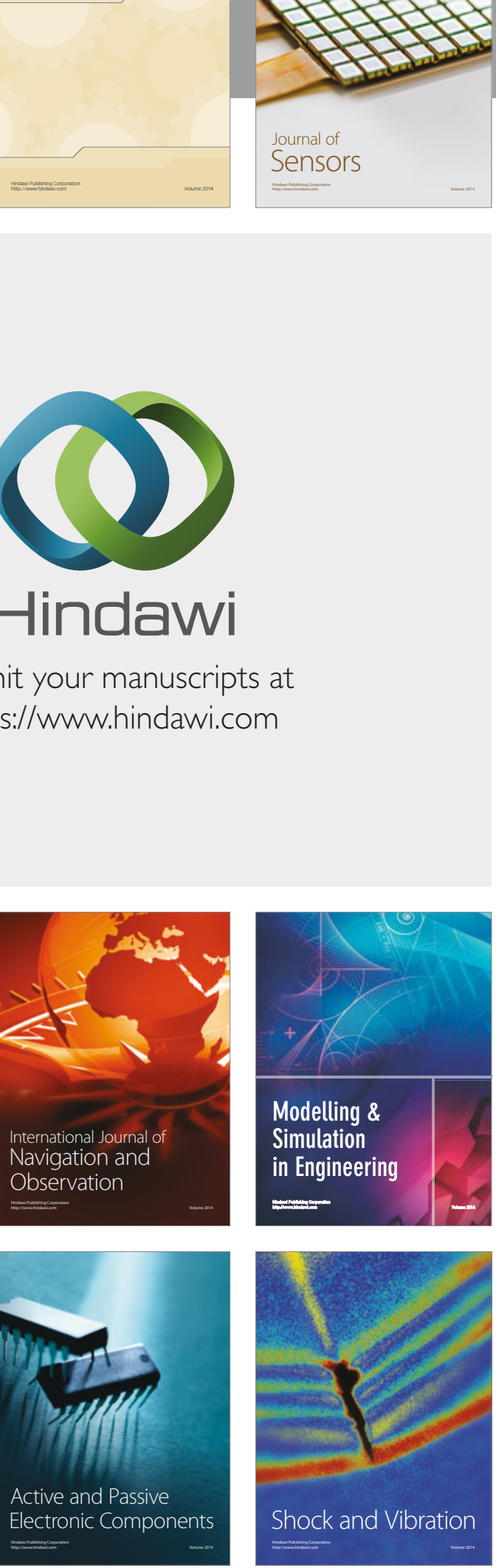
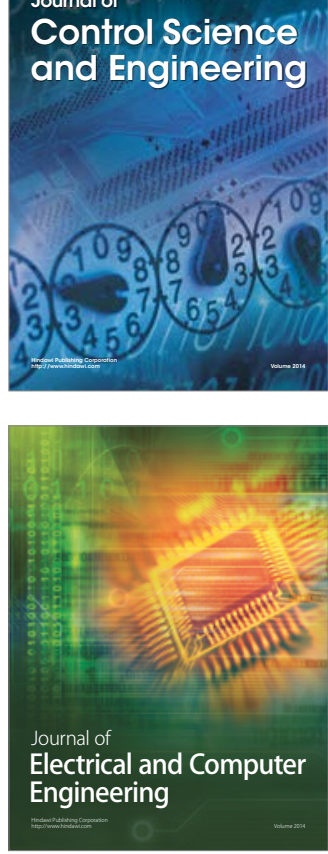

Distributed

Journal of

Control Science

and Engineering
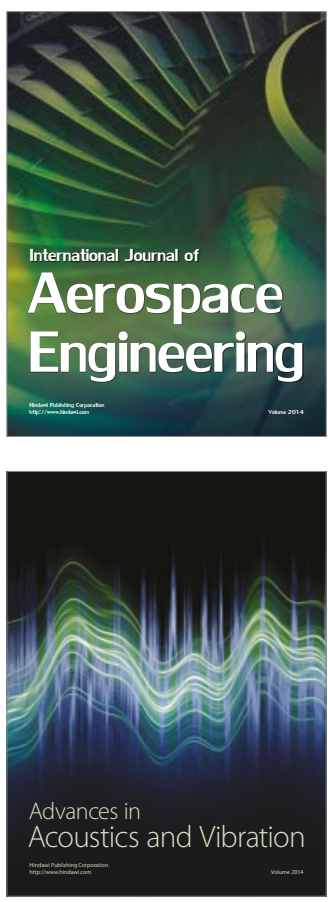

Sensor Networks 